\title{
iAWARE: Um modelo para Cuidado ubíquo de Pacientes com Transtornos de Ansiedade, Depressão e Estresse utilizando Gamificação e Biodata
}

\author{
Lucas Pfeiffer Salomão Dias \\ lucaspfsd@gmail.com \\ Universidade do Vale do Rio dos Sinos (UNISINOS) \\ São Leopoldo, RS
}

\begin{abstract}
Depression and anxiety are the most prevalent mental disorders, reaching million people worldwide. As they share many characteristics these two disorders can manifest themselves together. In addition, stress is one of the related factors with both depression and anxiety, being relevant in the analysis of these disorders. This paper proposes a model for ubiquitous care of patients with depression, anxiety and stress disorders (DASD) using gamification and biodata, called iAware. A/B tests evaluated with a clinical population the interaction engagement of the patient to the treatment provided by gamification. Based on the patient's history, the iAware monitors and applies interventions for the patient at the most appropriate time. Six patients used a prototype with a smartband for two weeks to evaluate iAware. Interactions with intervention stages were greater in patients who used iAware gamified. A more detailed analysis is still required. The evaluated patients got more occurrences of anxiety at home. The patients also filled out a survey based on the Technology Acceptance Model (TAM). TAM results show that patients have found the iAware useful in their anxiety treatment routines.
\end{abstract}

\section{KEYWORDS}

depression, anxiety, stress, gamification, biodata

\section{INTRODUÇÃO}

Este artigo apresenta um resumo dos resultados obtidos na dissertação de mestrado defendida no Programa de Pós-graduação em Computação Aplicada (PPGCA) na Universidade do Vale do Rio dos Sinos (UNISINOS) em 17/08/2018. O trabalho foi desenvolvido pelo primeiro autor, num período de 24 meses, sob a orientação do segundo autor.

Depressão e ansiedade são os transtornos mentais com maior prevalência na população mundial [15]. Somente a depressão atinge mais de 300 milhões de pessoas, representando $4.4 \%$ da população [15]. Por sua vez, a ansiedade atinge mais de 260 milhões de pessoas, representando $3.8 \%$ [15]. Estes dois transtornos podem se manifestar separadamente, porém, frequentemente manifestam-se em conjunto [13], uma vez que compartilham muitas características

In: I Concurso de Teses e Dissertações (CTD 2019), Rio de Janeiro, Brasil. Anais Estendidos do Simpósio Brasileiro de Sistemas Multimídia e Web (WebMedia). Porto Alegre: Sociedade Brasileira de Computação, 2019.

ISSN 2596-1683

\author{
Jorge Luis Victória Barbosa \\ jbarbosa@unisnos.br \\ Universidade do Vale do Rio dos Sinos (UNISINOS) \\ São Leopoldo, RS
}

[2]. Além disso, o estresse é um dos fatores relacionados com a depressão e ansiedade, sendo relevante na análise desses transtornos [12].

Em um contexto de $u$-Health para gerenciamento de TADE, os smartphones e wearables podem ser utilizados no apoio à prevenção e ao gerenciamento desses transtornos em função do seu uso discreto e a sua variada gama de sensores $[1,14]$. Estes dispositivos podem ser utilizados para monitorar comportamentos[5, 8], auxiliar a execução de tratamentos, ensinar técnicas de intervenção e coletar dados biológicos (biodata), como por exemplo, quantidade de passos, frequência cardíaca e qualidade do sono [10].

Este artigo propõe o iAware, um modelo para o apoio ao tratamento de TADE através de intervenções gamificadas, com suporte à biodata. A contribuição científica deste estudo está relacionada a duas principais lacunas que foram encontradas através dos trabalhos relacionados. A primeira consiste na avaliação da eficácia da gamificação no tratamento de TADE e a segunda na utilização de biodata para o acompanhamento do paciente.

O texto está organizado em seis seções. A seção 2 apresenta o modelo iAware, sua arquitetura e ontologia. Os aspectos de avaliação são apresentadas na seção 3. Os resultados são abordados na seção 4 e por fim na seção 5 , são apresentadas as conclusões e trabalhos futuros.

\section{MODELO}

O iAware é um modelo para cuidado ubíquo de pacientes com transtornos de ansiedade, depressão e estresse, que utiliza intervenções gamificadas e biodata. O modelo possui agentes específicos para coleta de biodata e outros dados de contexto, os quais aplicam intervenções e gamificação. A criação do iAware foi baseada em uma revisão bibliográfica envolvendo gamificação, jogos sérios e cuidado de depressão [7]. Nas próximas subseções serão apresentados a arquitetura, módulos, agentes, aplicações e a ontologia do iAware.

\subsection{Arquitetura}

A arquitetura do iAware é organizada em módulos, agentes, aplicações e ontologia. A Figura 1 apresenta a visão geral do iAware, mostrando seus componentes e como eles se comunicam. Os módulos são expostos em uma Web API que fornece ações para uso nos agentes e também nas aplicações. Por ter uma estrutura modular, novos recursos ou atribuições podem ser aplicadas ao iAware a fim de estender suas funcionalidades. Os agentes são independentes, reagem ao contexto e se comunicam pelo uso de ações que são geridas pelo módulo de ações. As aplicações se comunicam com 
os módulos através da API baseada no protocolo HTTP a fim de mostrar os recursos e atividades específicas a cada tipo de usuário, paciente ou psicólogo. A ontologia apresenta o conhecimento relacionado ao domínio no tratamento de TADE. Este conhecimento foi estruturado em um trabalho conjunto com psicólogos que participaram da pesquisa.

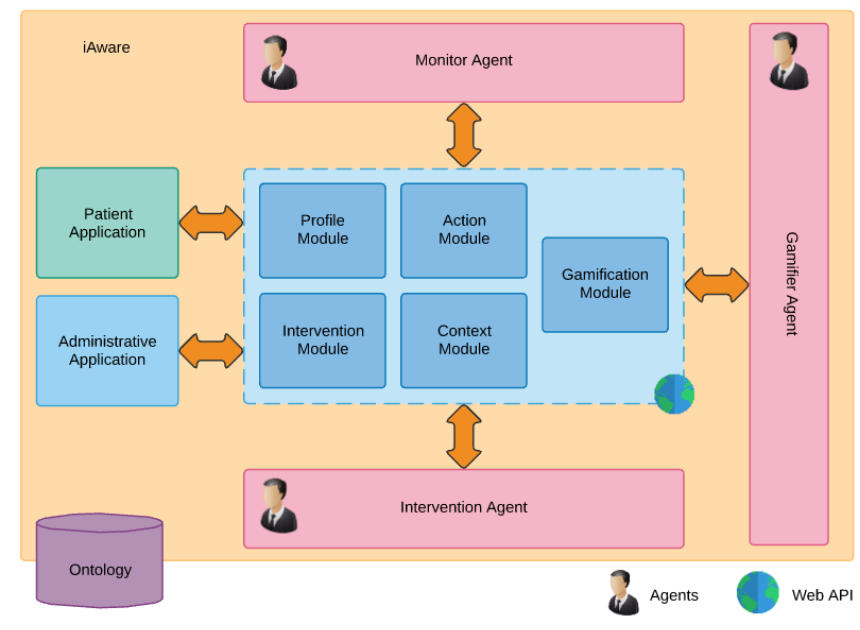

Figure 1: Visão Geral do Modelo

\subsection{Módulos}

O iAware possui cinco módulos, sendo eles Perfil, Ação, Gamificação, Intervenção e Contexto. Os módulos de Ação e Contexto são independentes, enquanto que os módulos de Gamificação, Intervenção e Perfil possuem uma relação de dependência. Esta dependência existe uma vez que para a gamificação ocorrer é necessário que haja uma intervenção, e para saber qual intervenção aplicar, é necessário saber o perfil do paciente, que receberá a intervenção.

\subsection{Agentes}

O iAware tem ações autônomas em coletas de dados de contexto, aplicação de intervenções e na gamificação de suas atividades. Essas ações acontecem continuamente e simultaneamente adaptando-se conforme a experiência do usuário. A Figura 2 apresenta uma visão geral dos agentes, onde é possível ver a interação que ocorre entre os agentes Monitorador, Interventor e Gamificador.

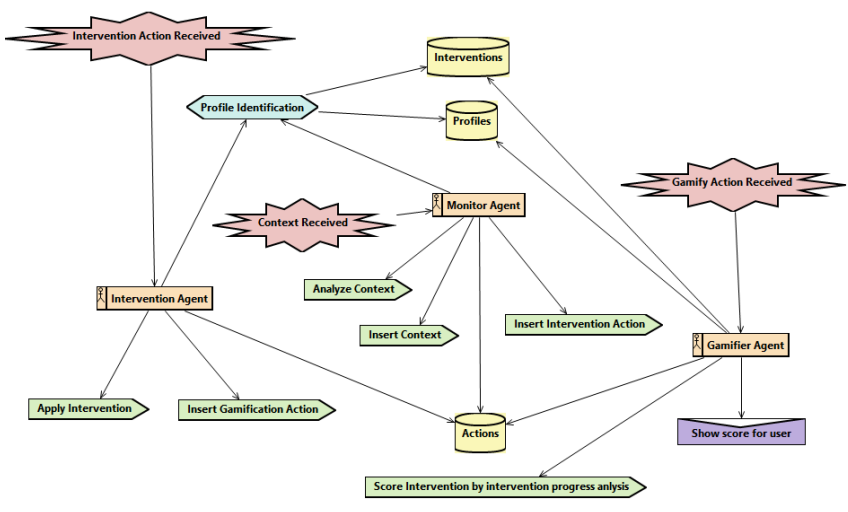

Figure 2: Diagrama dos agentes do iAware

\subsection{Ontologia do iAware}

A ontologia do iAware tem por finalidade representar o conhecimento relacionado ao domínio do modelo proposto. A Figura 3 apresenta a ontologia proposta no iAware. Uma das vantagens do uso de ontologias, é estruturar conhecimentos que depois serão compartilhados entre os agentes e também com outros sistemas. A ontologia é um componente básico para o modelo, uma vez que ela representa o conhecimento a respeito do domínio de cuidado ubíquo de pacientes com TADE, de forma que os agentes compartilhem o mesmo entendimento sobre os conceitos expressos nessa representação de conhecimento.

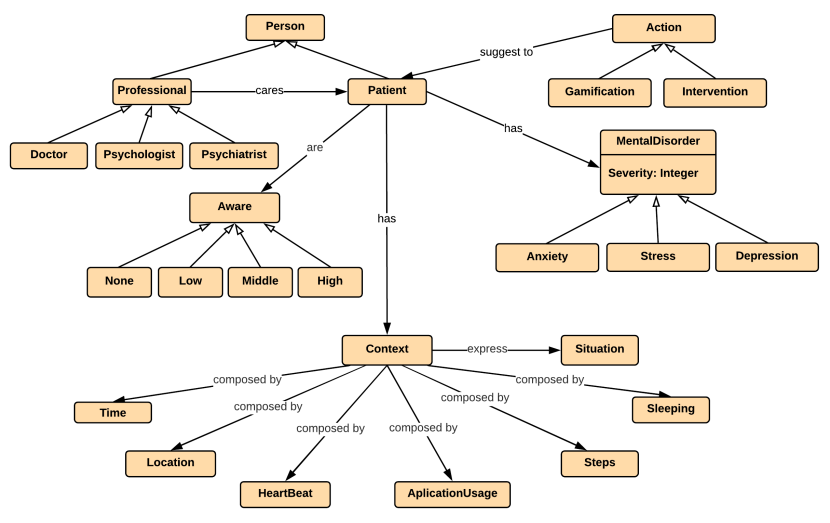

Figure 3: Ontologia do iAware

\subsection{Aplicação do Paciente}

A Aplicação do Paciente faz sugestões de intervenção ao paciente por meio de notificações, e permite que ele realize tais intervenções. Esta aplicação também apresenta recursos de gamificação como a pontuação, nível de consciência do tratamento e as recompensas. Para garantir que a intervenção seja aplicada no momento mais apropriado, a aplicação do paciente deve estar sempre próxima a ele, para que ela consiga monitorá-lo, notificá-lo, aplicar a intervenção e engajá-lo pelo uso da gamificação. Portanto, entende-se como Aplicação do Paciente um ou mais dispositivos que garantam estas quatro características descritas. 


\subsection{Aplicação de Administração}

A Aplicação de Administração apresenta a quantidade de intervenções realizadas e quais intervenções foram mais eficazes para o paciente. Por meio da medição da pontuação da intervenção realizada pelo usuário, o profissional da saúde pode ajustar o tratamento do paciente e estar continuamente atualizando o perfil do paciente, podendo alterar as características anteriormente configuradas. O sistema possui duas formas de feedback do comportamento do usuário, uma baseada no histórico de contextos $[3,4,11]$ e outra nas características do perfil do paciente. Diferente da Aplicação do Paciente, a Aplicação de Administração é utilizada apenas quando o profissional da saúde achar conveniente ter acesso aos dados do paciente ou desejar atualizar o seu perfil.

\section{ASPECTOS DE AVALIAÇÃO}

O iAware foi avaliado através da aplicação de um questionário baseado no Modelo de Aceitação de Tecnologia (TAM). A satisfação do usuário é medida em duas categorias: utilidade percebida e facilidade de uso percebida [6]. O questionário é composto por itens de Likert [9] de cinco níveis que são, 'discordo totalmente', 'discordo parcialmente', 'indiferente', 'concordo parcialmente' e 'concordo totalmente'.

Desde o início do estudo, foi mantido contato com pesquisadores do PPG em Psicologia da instituição acadêmica onde o estudo foi elaborado, os quais fizeram parte da equipe de pesquisa, auxiliando no estudo e colaborando na identificação de profissionais que poderiam participar do experimento. A avaliação foi aprovada pelo comitê de ética da instituição onde o estudo foi desenvolvido e possui o registro (CAAE 90200318.9.0000.5344) na Plataforma Brasil (http : //plataformabrasil.saude.gov.br).

Seis pacientes foram selecionados e separados aleatoriamente em dois grupos iguais, onde um dos grupos utilizou o iAware gamificado e o outro o iAware não gamificado. Ambos os grupos utilizaram o iAware instalado em seus smartphones durante, no máximo, duas semanas. Após as duas semanas de utilização do iAware, o paciente voluntário era convidado a responder o questionário TAM e o psicólogo participava de uma entrevista semiestruturada individual para relatar suas percepções da utilização do iAware no tratamento do seu paciente.

\section{RESULTADOS}

Seis pacientes utilizaram o iAware, sendo que um deles não se adaptou a utilização da pulseira e por isso completou apenas uma semana das duas previstas no experimento. Os resultados foram divididos em seis subseções, sendo elas avaliação da gamificação, lugares onde houveram ocorrências de ansiedade, turnos onde houve maior incidência de ansiedade, comportamento quando houve ansiedade, avaliação das técnicas da TCC e avaliação TAM. Os pacientes que utilizaram o iAware gamificado serão identificados como P1, P2 e P3, enquanto os que utilizaram a versão não gamificada serão identificados como P4, P5 e P6.

\subsection{Avaliação da Gamificação}

Ao final da utilização do protótipo a interação dos pacientes no iAware foi coletada e analisada. A interação possuía três etapas, sendo elas a notificação, a realização e a pontuação da atividade a ser realizada pelo paciente.

Os pacientes que utilizaram o iAware gamificado tiveram uma homogeneidade nas interações, realizando as três etapas. No entanto estes pacientes tiveram apenas uma ocorrência de ansiedade durante o experimento, isso ocorreu devido a eles terem diferentes graus de ansiedade e devido ao curto tempo de experimento. Já os pacientes que não utilizaram a gamificação tiveram mais ocorrências de ansiedade durante o experimento, porém não realizaram as três etapas em todas as interações. Este resultado apresenta indícios da falta de engajamento dos pacientes, que não utilizaram a gamificação, na realização de todas as etapas da interação. No entanto devido ao grupo de pacientes avaliados ser pequeno e durante o período do experimento ter ocorrido poucas incidências de ansiedade do grupo que utilizou a gamificação, não é possível afirmar que a completude das etapas de interação da atividade foi resultado da utilização de recursos da gamificação.

\subsection{Lugares onde ocorreram ansiedade}

Esta análise visou identificar no grupo de teste em quais lugares ocorreram momentos de ansiedade. Os pacientes podiam identificar os locais onde estavam por meio de seis categorias, sendo as opções: casa, trabalho, escola/faculdade, casa de parentes, loja/shopping e outros. Quando não identificado um local ele era automaticamente considerado "outros".

A casa foi o único local onde ocorreu ansiedade para os pacientes P1 e P5, durante as duas semanas de utilização do iAware. Já para os pacientes $\mathrm{P} 2$ e $\mathrm{P} 3$ a ansiedade se manifestou apenas na casa de parentes. O paciente $\mathrm{P} 6$ relatou ansiedade em casa e em "outros locais". O paciente $\mathrm{P} 4$ foi o que relatou ansiedade em uma maior diversidade de locais, sendo eles a casa, escola/faculdade e "outros".

\subsection{Comportamento quando houve ansiedade}

Com o intuito de entender o comportamento dos pacientes durante os momentos em que se encontravam com ansiedade, foram analisadas a quantidade de passos, média dos batimentos cardíacos por minuto (BPM) e batimento cardíaco máximo capturados durante o turno em que ocorreu a ansiedade. A média de BPM variou entre 66,61 à 93,01. Já o BPM máximo variou de 92 à 165, sendo que os maiores valores de BPM Máximo 165 (P6) e 129 (P2) tiveram uma média intermediária de 82,07 e 80,94 respectivamente. Os pacientes $\mathrm{P} 4$ e $\mathrm{P} 3$ foram os que relataram o nível máximo de ansiedade percebida, tendo BPM Máximo de 108 e média de 83,06 BPM e BPM Máximo de 112 e média de 66,61 BPM, respectivamente.

Dos gatilhos disparados através da Bio Observação, os pacientes P2 e P4 tiveram alteração de batimentos cardíacos durante a madrugada, no entanto, seus níveis de ansiedade foram relatados como baixos. Os psicólogos participantes da pesquisa acreditam que o disparo deste gatilho de fato foi em virtude de ansiedade, mas não assim interpretada pelos pacientes. Os psicólogos creem que se a pergunta mencionasse dificuldades para dormir ao invés de ansiedade percebida, o resultado poderia ter sido mais assertivo, pois a insônia é uma característica da ansiedade, mesmo que não seja assim interpretada pelo paciente.

Ocorreram dois gatilhos disparados pela Bio Observação do paciente $\mathrm{P} 2$, o qual havia realizado exercícios e depois repousado. A 
atividade física foi identificada ao analisar que 5 minutos antes do gatilho da Bio Observação ter ocorrido, o paciente P2 estava elevando a quantidade de passos em média de 100 por minuto, o que já é considerado atividade física. Esse falso positivo ocorreu devido a janela de observação de 5 minutos não ser suficiente para que o ritmo cardíaco voltasse ao normal. O paciente informou o nível mínimo, confirmando assim a ausência de ansiedade.

\subsection{Avaliação das Técnicas da TCC}

As intervenções ofertadas para os pacientes que utilizaram o iAware são provenientes da Terapia Cognitivo-Comportamental (TCC) e foram as mesmas para os dois grupos, com e sem gamificação. A técnica de Respiração obteve 7 ocorrências, seguida de Relaxamento Muscular com 4 ocorrências e Mindfulness com 3 ocorrências.

Outra avaliação das técnicas foi realizada com a média da pontuação dada pelos pacientes. Ao finalizar o vídeo com a técnica, o paciente podia avaliá-la com uma nota de 1 a 5 pontos. A técnica de mindfulness foi a que recebeu a maior média de pontuação, 3,5 pontos. Em segundo lugar ficou a técnica de respiração que obteve 3 pontos e por fim a técnica de relaxamento muscular, com 2,33 pontos.

\subsection{Avaliação TAM}

As respostas de cada categoria, utilidade e usabilidade, no geral foram positivas. Nos resultados das médias dos pacientes através do TAM, para afirmações relacionadas à utilidade do iAware 33\% dos pacientes concordaram totalmente que o aplicativo é útil a sua rotina de tratamento, $50 \%$ concordaram parcialmente e $17 \%$ foram indiferentes. Sobre a usabilidade do iAware $83 \%$ dos pacientes concordaram totalmente que o iAware é fácil de usar e $17 \%$ discordaram parcialmente.

\section{CONCLUSÃO}

Os resultados obtidos através dos experimentos indicam que a utilização do iAware para o apoio ao tratamento de ansiedade é possível e útil ao paciente, porém, ainda é necessária uma avaliação com um grupo maior de pacientes para uma análise mais precisa. A análise da gamificação foi realizada por meio da interação do usuário durante as atividades de intervenção. Apenas os pacientes que utilizaram o iAware gamificado completaram todas as etapas. Essa avaliação mostra indícios que a gamificação engajou os pacientes a completarem as três etapas da intera-ção no iAware. A avaliação dos lugares onde houve ocorrência de ansiedade aponta que os pacientes tiveram a maior incidência de ansiedade em sua casa, sugerindo que este local poderia contar com recursos de um ambiente inteligente para um aperfeiçoamento no acompanhamento e tratamento da ansiedade.

Através da análise do comportamento dos pacientes foram identificados possíveis momentos de ansiedade de dois pacientes durante a madrugada. No entanto, os pacientes identificaram este momento com um nível baixo de ansiedade. Foi questionado aos psicólogos o que poderia ter causado essa informação de um nível baixo de ansiedade percebida, tendo em vista que houve um aumento da média dos batimentos cardíacos. Os psicólogos inclinaram-se a acreditar que houve um momento de ansiedade e creem que se a pergunta do iAware mencionasse dificuldades ao dormir, ao invés de ansiedade percebida, a resposta dos pacientes poderia ter sido mais assertiva. Assim, este estudo apresenta indícios da possibilidade de utilizar biodata para o acompanhamento de pacientes com transtorno de ansiedade.

\section{ACKNOWLEDGMENTS}

Os autores agradecem à Fundação de Amparo à Pesquisa do Estado do Rio Grande do Sul (FAPERGS), à Coordenação de Aperfeiçoamento de Pessoal de Nível Superior - Brasil (CAPES) - Código de Financiamento 001, ao Conselho Nacional de Desenvolvimento Científico e Tecnológico (CNPq) e à Universidade do Vale do Rio dos Sinos (Unisinos) pelo apoio ao desenvolvimento desse trabalho.

\section{REFERENCES}

[1] Aino Ahtinen, Elina Mattila, Pasi Välkkynen, Kirsikka Kaipainen, Toni Vanhala, Miikka Ermes, Essi Sairanen, Tero Myllymäki, and Raimo Lappalainen. 2013. Mobile mental wellness training for stress management: Feasibility and design implications based on a one-month field study. Journal of Medical Internet Research 1 (2013), e11.

[2] G. Andrews, D. P. Goldberg, R. F. Krueger, W. T. Carpenter, S. E. Hyman, P. Sachdev, and D. S. Pine. 2009. Exploring the feasibility of a meta-structure for DSM-V and ICD-11: could it improve utility and validity?: Paper 1 of 7 of the thematic section: 'A proposal for a meta-structure for DSM-V and ICD11'. Psychological Medicine 39, 12 (2009), 1993-2000. https://doi.org/10.1017/ S0033291709990250

[3] Jorge Barbosa, João Tavares, Ismael Cardoso, Bruno Alves, and Bruno Martini. 2018. TrailCare: An indoor and outdoor Context-aware system to assist wheelchair users. International fournal of Human-Computer Studies 116 (2018), 1 - 14. https://doi.org/10.1016/j.ijhcs.2018.04.001

[4] Jorge Luis Victória Barbosa, Cládio Martins, Laerte Kerber Franco, and Débora Nice Ferrari Barbosa. 2016. TrailTrade: A model for trail-aware commerce support. Computers in Industry 80 (2016), 43-53. https://doi.org/10.1016/j.compind.2016. 04.006

[5] Paulo Chaló, Anabela Pereira, Patrícia Batista, and Luis Sancho. 2017. Brief Biofeedback Intervention on Anxious Freshman University Students. Applied Psychophysiology and Biofeedback 42, 3 (01 Sep 2017), 163-168. https://doi.org/ 10.1007/s10484-017-9361-5

[6] Fred D Davis. 1989. Perceived usefulness, perceived ease of use, and user acceptance of information technology. MIS quarterly 1, 1 (1989), 319-340.

[7] Lucas Pfeiffer Salomão Dias, Jorge Luis Victória Barbosa, and Henrique Damasceno Vianna. 2018. Gamification and serious games in depression care: A systematic mapping study. Telematics and Informatics 35, 1 (2018), $213-224$. https://doi.org/10.1016/j.tele.2017.11.002

[8] Gregg Henriques, Steven Keffer, Craig Abrahamson, and S. Jeanne Horst. 2011. Exploring the Effectiveness of a Computer-Based Heart Rate Variability Biofeedback Program in Reducing Anxiety in College Students. Applied Psychophysiology and Biofeedback 36, 2 (01 Jun 2011), 101-112. https://doi.org/10.1007/ s10484-011-9151-4

[9] Rensis Likert. 1932. A technique for the measurement of attitudes. Number 140 in 1. New York University, New York

[10] L. Mertz. 2016. Convergence Revolution Comes to Wearables: Multiple Advances are Taking Biosensor Networks to the Next Level in Health Care. IEEE Pulse 7, 1 (Jan 2016), 13-17. https://doi.org/10.1109/MPUL.2015.2498475

[11] João H. Rosa, Jorge L. V. Barbosa, Marcos Kich, and Lucas Brito. 2015. A MultiTemporal Context-aware System for Competences Management. International Journal of Artificial Intelligence in Education 25, 4 (01 Dec 2015), 455-492. https: //doi.org/10.1007/s40593-015-0047-y

[12] Jessica Schleider, Elizabeth D. Krause, and Jane Gillham. 2014. Sequential Comorbidity of Anxiety and Depression in Youth: Present Knowledge and Future Directions. Current Psychiatry Reviews 10 (01 2014), 75-87.

[13] Cyd C. Strauss, Cynthia G. Last, Michel Hersen, and Alan E. Kazdin. 1988. Association between anxiety and depression in children and adolescents with anxiety disorders. Journal of Abnormal Child Psychology 16, 1 (01 Feb 1988), 57-68. https://doi.org/10.1007/BF00910500

[14] Fabian Wahle, Tobias Kowatsch, Elgar Fleisch, Michael Rufer, and Steffi Weidt. 2016. Mobile Sensing and Support for People With Depression: A Pilot Trial in the Wild. FMIR mHealth and uHealth 4, 3 (Sep 2016), e111.

[15] Geneva: World Health Organization WHO. 2017. Depression and Other Common Mental Disorders Global Health Estimates. Licence: CC BY-NC-SA 3.0 IGO. 